\title{
Various OCT Segmentation and Classification Techniques
}

\author{
Neenu Joy ${ }^{1}$, Dr.Anishin Raj $\mathbf{M M}^{2}$ \\ ${ }^{1}$ Mtech Student (CSE), Viswajyothi College of Engineering and Technology, Vazhakulam, Kerala, \\ neenuj89@gmail.com \\ ${ }^{2}$ Associate Professor (CSE), Viswajyothi College of Engineering and Technology, Vazhakulam, Kerala, \\ anishinraj@vjcet.org
}

\begin{abstract}
Optical coherence tomography is a non-intrusive method for the image. OCT is a well-known modality of detecting and inventing ocular disease on time. From an ophthalmological viewpoint, any variations in the thickness of retinal layers differ in particular shape and texture and any discrepancies in normal population can be interpreted as a sign of the rare ocular condition. Since the study of ophthalmic images generally suffers from time complexity and is susceptible to subjective inaccuracies, there has been great interest in the automatic assessment of these images at a certain point in recent years. This paper describes different methods for the analysis of OCT images and their comparison. In this paper various models of image segmentation and classification are discussed.
\end{abstract}

Key words- Biomarker, Classification Techniques, CNN, OCT, Segmentation Techniques, Supervised, Unsupervised.

\section{INTRODUCTION}

Optical coherence tomography is a non-intrusive method for image. OCT is a well-known modality of detecting and inventing ocular disease on time. OCT images from the retina have similar characteristics but in particular the variety of great features relies on normal $\backslash$ abnormal eye circumstance. From an ophthalmological viewpoint, any variations in the thickness of retinal layers differ in particular shape and texture and any discrepancies in normal population can be interpreted as a sign of rare ocular condition. Since the study of ophthalmic images generally suffers from time complexity and is susceptible to subjective inaccuracies, there has been great interest in the automatic assessment of these images at a certain point in recent years.

The section in this paper is organized as; in section 2, the basics of OCT analysis methods are discussed. Section 3 deals with the survey on some OCT segmentation and classification techniques. Section 4 and Section 5 give some discussion and conclude this paper.

\section{OCT ANALYSIS METHODS}

OCT analyzes are either supervised or unsupervised. We do not need to supervise the model in unsupervised training.

Rather, allowing the model to work alone to discover knowledge. In Supervised learning, labeled training data is used for testing, i.e. data is marked with the correct outcome. Supervised learning algorithms analyze the training data and forecast outcomes for unpredicted data when a new collection of data is fed into the system. Large scale annotation is costly or infeasible for supervised learning. It also restricts detection to known groups of diseases, and delays the identification of new markers in general. On the contrary, unsupervised discovery of abnormal candidates and subsequent data-based marker classification provide the opportunity for balanced disease segregation and recognition of new risk factors. Unsupervised research will expand our understanding of the original pathway of disease physiology. Unsupervised learning are divided into

- Clustering: to discover similarities and differences. It categorizes related things together.

- Dimensionality reduction: to find a improved (less complex) illustration of the data

Some of the recently published papers for OCT classification methodologies are analyzed and compared. Comparatives study including supervised learning methods, unsupervised learning methods using different networks.

\section{LITERATURE SURVEY}

Technique for unsupervised spotting of marker candidates in OCT is suggested in 1. OCT images are pre-processed. Boundary layers of the retina are established and flattened during preprocessing to create a $2 \mathrm{D}$ patch. Uses this $2 \mathrm{D}$ patch to train DDAE. This de-marks the abnormal candidates from normal samples in retinal SD-OCT in the light of the characteristics acquired by DDAE.One class SVM is utilized to distinguish anomalies in new data. SVM differentiates anomalous candidates from normal tissue in retinal SD-OCT based on the DDAE's learned characteristics. Frequently occurring anomalies are categorised using K- mean clustering algorithm, and evaluating their relation to ailment. Clustering of anomalies 
helps to categories samples into healthy, early AMD- and late AMD cases.

An approach for the automatic retinal layers segmentation in spectral-domain OCT images is suggested in 2. Here the segmentation problem is changed into the graph-theoretic problem where a minimum closed set is calculated in a geometric graph. The graph is constructed in order to represent the feasibility constraints and cost function, respectively, by organizing the graph and vertex weight. The minimal-cost closed set is located by locating a minimum s-t cut in a graph which is strongly related. In the first step, the surfaces close to the RPE became a flat plane by flattening the image. Flattening the 3-D OCT helps (1) to allow a small sized image in segmentation. (2) Provide a more regular outline for segmentation and 3) to provide a better idea and match to medical practice. Flattening the image decreases the size of the image in axial direction, which helps to tackle the intra-retinal layer segmentation technique's time and memory and requirements. To the flattened and truncated spectral images, anisotropic diffusion method is applied. Then the surface is segmented into 7 groups. Here the surfaces 1, 6, and 7were segmented all together, accompanied via the immediate segmentation of surfaces 2 , 3,4 , and 5 .

The method suggested in 3 is for training a CNN that doesn't depend upon any categorized data. Unlabeled images create surrogate tasks automatically and CNN makes use of these surrogate tasks. Surrogate images are generated by data augmentation methods. The substitute task is intended to defer normal features which can be explanatory and sturdy to normal deviations in the data. Random transformations are applied to a 'seed 'image to simulate variants. Transformations carried out to seed image are scaling, rotation, translation, color variant, and contrast variant. Seed image and its converted variations represent a surrogate class. In this strategy, to make class only a one seeding pattern is needed. Then the CNN called Exemplar$\mathrm{CNN}$ is quantified to distinguish surrogate classes. The representation found out by the Exemplar-CNN is discriminative, whilst also invariant to modifications. Because of these properties, Exemplar-CNN is used for various vision tasks. The characteristic learned by the Exemplar-CNN performs properly on: object classification and descriptor matching. This algorithm is substantially less responsive to the extent of the transformation applied. In order to serve other programs of the learned capabilities, different transformations can be added to this technique.

A method for the automatic classification of 3-D retinal fluid is mentioned in 4. It addresses the class imbalance issue of SEAD technique. SD-OCT images are enhanced using pre-processing strategies to improve the SNR. Preprocessing consists of a recursive anisotropic diffusion filter and brightness curve transform so that it will diminish the noise and improve the abnormality regions. Preprocessed images are segmented into eleven surfaces. Hierarchical segmentation starts from easily noticeable ones and ends with the most delicate interfaces. To smooth the detected surfaces, a thin plate spline is applied. A Stratified sampling based voxel category approach is used. In this, a stratified pattern is built by means of organizing the entire region into strata. The classification is based on few foregone uniqueness of the regions. The strata have to be jointly exclusive and together exhaustive. i.e., 1 is assigned to every point inside the sample region and simplest to 1 stratum. During the training phase, features like structural, positional and textural information are calculated for each sample point. In the testing stage, for each voxel in retina layers the same sets of features were extracted. Then as the probability of being a SEAD, each voxel was assigned with an average label of the $\mathrm{k}$ nearest neighbors. Each voxel has a probability between 0 and 1 .

An object detection algorithm known as Faster R-CNN is suggested in 5. A deep FCN called RPN and a Fast R-CNN detector are its two modules. RPN proposes regions and the proposed regions are utilized by Fast R-CNN. The whole device is a single, united network for object identification. Object boundaries and scores at each location are predicted by RPN. The RPN is taught to produce top notch location proposals. An arbitrary sized input is given to a Region Proposal Network (RPN) and a set of object proposals along with objectness score is generated. The CNNs are taught end-to-end by means of the R-CNN method to group the proposal regions into background or object categories. Aside from refining by bounding box, regression, R-CNN no longer predicts object boundaries. It performs as a classifier. The overall execution of the region proposal module defines its accuracy. The Fast R-CNN module is guided by the RPN module by pointing where to look. A convolutional feature map is created from CNN by providing input images to it. The Regions of proposals are identified from the feature map and twist them into squares. To feed the fully connected layer, reshape the region proposals into a fixed size by utilizing a RoI pooling layer. Class of the proposed area and offset values for the bounding box from the RoI feature vector, is anticipated by using a softmax layer.

Automatic recognition of four retinal layer borders is suggested by 6 . In this methodology first a surmised area of three reference layers is generated. Afterwards utilizes these to bind the actual layer's search space. Followed by modeling the issue as a graph, Dijkstra's shortest path algorithm is applied. By using a canny edge detection algorithm, edge pixels are detected. Candidate pixels are selected by filtering edge pixels based on the boundary position and intensity gradient. Based on the number of pixels candidate pixels are grouped to form a pixel-group. Each pixel group's end pixels form a node in the graph. The edge weight connecting nodes is resolved utilizing pixel distance, slope similarity to a reference and non-associativity of the layers. In this way the distorting impacts of the disease in the boundary determination can be conquered. The shortest path between the two nodes (starting and ending nodes) are computed by using Dijkstra's algorithm. 
From the candidate pixels list, the shortest path corresponding to the nodes in pixel groups is extracted and using extrapolation the remaining non-connected pixels are joined. To remove remaining noise, Gaussian smoothing is applied to the boundary as the final step. To recognize retina and two basic sorts of macular pathologies such as AMD and DME 7 proposed a CAD framework dependent on a multi-scale convolutional mixture of expert ensemble models (MCME). The proposed framework comprises two primary steps, a pre-processing and categorization step. In the pre-processing step, the retinal distortions are removed by utilizing a graph based curvature and correction algorithm. This step helps to generate a set of standard region (volume) of interests (ROIs/VOIs). Preprocessing steps includes 1. Normalizations: To get a unique view for OCT image, 2) Image flattening and cropping: for the retinal smoothing by utilizing a graph based geometry curvature correction algorithm. 3) ROI and VOI generation, augmentation strategy. 4) Multi-scale spatial decomposition: before sustaining macular OCT to convolutional ensemble models, multi-scale spatial pyramid decomposition is applied. In the classification step, a robust image-based classifier based on a mixture of CNN experts is used. The expert and gating system are developed by CNNs. The scaledependent modules utilized in MCME empower the model to have a multi-scale perception of inputs. Last output of the ME is a weighted sum of the experts 'output, where the weight vector is the yield of the gating system.

A Fully Convolutional Network is prepared for image partition is presented in 8 . The key thought in FCN-based techniques is that they take in a mapping from pixels to pixels, without removing the region proposals. The FCN network pipeline is an expansion of the old style CNN. The FCN takes an image with a discretionary size and creates a segmented image with a similar size. In spite of the fact that this is the situation, the size of the output of FCNs relies upon the input size as opposed to continually creating a fixed-size output. In this manner, these sorts of systems are generally utilised for local task as opposed to global task (i.e., semantic segmentation or object detection rather than object classification). The creators start by altering popular architectures (Alex Net, VGG16, Google Net) to have a non fixed size input while supplanting all the fully connected layers by convolutional layers. Since the system delivers a few element maps with little sizes and dense representations, an Upsampling is important to make an output with a similar size than the input. Essentially, it comprises a convolutional layer with a stride inferior to 1 . It is normally called deconvolution since it makes an output with a bigger size than the input. Thus, the system is prepared utilising a pixelwise loss. In addition they have included skip architecture in the system to consolidate significant level feature map representations with specific and dense ones at the top of the network.

A technique to accomplish the classification of surrogates of the original OCT images by utilising the trained CNN model is proposed in 9. OCT images have speckle noise and to reduce that denoising is applied. In this paper sparse representation based strategy is used for denoising. OCT image's background doesn't hold valuable data, so to confine the scope of the consequent operations; a mask of image is separated. Binary image is generated by applying Thresholding on the denoised image and Morphological dilation is applied to binary image to generate the mask. To produce a ton of surrogate image, the denoised image together with the mask is utilised. These surrogated images are then utilised to prepare the CNN model. The reasons for producing surrogate image are to expand the information and diminish the complexity of the seed image. Prior to training and testing the $\mathrm{CNN}$ mode, normalisation of contrast and brightening are performed on the surrogate image. Finally the average output from the prepared CNN model on the surrogate image determines the forecast for a test image. Only one CNN model is used in this work. The average output from the prepared model on surrogate image of every class accomplishes the ensemble.

For retinal OCT classification, a novel lesion aware CNN (LACNN) technique is introduced in 10. In this proposed technique, retinal lesions inside OCTs are used to direct the CNN to accomplish exact classification. The lesion attention module proposed by LCNN, protects the significant structures in OCT images and also improves the features from local lesion-related regions. The LACNN is consisting of lesion-attention modules, convolutional layers, and pooling layers. To recognize different sorts of macular lesions, a lesion detection network (LDN) is developed. The LDN is a CNN model which comprises convolution blocks, local contrast processing blocks, and deconvolution blocks. It is for the remarkable lesion identification and makes the attention maps of lesions. The attention maps empower the recommended LACNN model to concentrate more on the legitimate data. The identified attention maps are used to exquisitely weight the convolutional include classification network map. In every lesion-attention module, the data sources are the LDN's attention map and feature maps from previous convolutional layers and the output are lesion aware feature maps. The classification network uses the local lesion associated data to accomplish productive and exact OCT categorisation.

A novel methodology for anomaly discovery dependent on estimates of epistemic uncertainty from a Bayesian U-Net is proposed in 11. Bayesian U-net are at first prepared for segmenting healthy subjects using weak labels generated using segmentation based on graphs. Uncertainty estimates and segmentation maps are the output of the Bayesian network. Since the model is for segmenting abnormalities, uncertainty estimate from the network is used. To improve pixel-level epistemic uncertainty estimates, Monte Carlo dropout is applied to this model. As a post-handling step to alter the uncertainty maps into reduced segmentation of abnormalities, a majority-ray-casting is applied. This strategy reduces the disparity between the irregularities and the outline of layers dependent on the presumption that abnormality in OCT are reduced and not layered. 


\section{DISCUSSION}

This paper deals with various OCT segmentation and classification techniques. Each method has its own pros and cons. The summary of each methods advantage and disadvantage is given below.

Table 1: Comparison of Various OCT Segmentation and Classification Techniques.

\begin{tabular}{|c|c|c|c|}
\hline References & Techniques & Advantages & $\begin{array}{l}\text { Disadvanta } \\
\text { ges }\end{array}$ \\
\hline 1 & $\begin{array}{c}\text { Segmentatio } \\
\mathrm{n} \text { and } \\
\text { classification } \\
\text { of OCT } \\
\text { using } \\
\text { DDAE. }\end{array}$ & $\begin{array}{l}\text { 1. Without } \\
\text { constrai } \\
\text { nts to } \\
\text { prior } \\
\text { definitio } \\
\text { n, able } \\
\text { to detect } \\
\text { anomali } \\
\text { es in } \\
\text { new } \\
\text { volume } \\
\text { Meanin } \\
\text { gful } \\
\text { structure } \\
\text { in the } \\
\text { data is } \\
\text { revealed } \\
\text { by Data } \\
\text { driven } \\
\text { learning } \\
\text {. } \\
\text { Identifie } \\
\text { s stable } \\
\text { and } \\
\text { replicabl } \\
\text { e } \\
\text { anomaly } \\
\text { categori } \\
\text { es. }\end{array}$ & $\begin{array}{c}\text { 1. Relies } \\
\text { on } \\
\text { multi- } \\
\text { scale } \\
\text { deep } \\
\text { denoisin } \\
\text { g } \\
\text { encoder } \\
\text { s. }\end{array}$ \\
\hline 2 & $\begin{array}{c}\text { Graph based } \\
\text { segmentatio } \\
\text { n of OCT. }\end{array}$ & $\begin{array}{l}\text { 1. Method is } \\
\text { ideal for the } \\
\text { cost } \\
\text { function. } \\
\text { 2. Varying } \\
\text { constraints } \\
\text { and regional } \\
\text { information } \\
\text { can be } \\
\text { incorporated. }\end{array}$ & $\begin{array}{c}\text { 1. At the } \\
\text { point when } \\
\text { two } \\
\text { boundaries } \\
\text { are located } \\
\text { for a surface, } \\
\text { then the } \\
\text { transition } \\
\text { between the } \\
\text { two limits is } \\
\text { considered }\end{array}$ \\
\hline
\end{tabular}

\begin{tabular}{|c|c|c|c|}
\hline & & & as boundary. \\
\hline 3 & $\begin{array}{l}\text { Classificatio } \\
\text { n of OCT } \\
\text { using } \\
\text { Exemplar } \\
\text { CNN. }\end{array}$ & $\begin{array}{c}1 . \\
\text { Classificatio } \\
\mathrm{n} \text { accuracy is } \\
\text { improved } \\
\text { when } \\
\text { compared } \\
\text { with } \\
\text { previous } \\
\text { unsupervised } \\
\text { methods } \\
\text { 2. Adaptable } \\
\text { to extend } \\
\text { with other } \\
\text { transformati } \\
\text { ons. } \\
\text { 3. } \\
\text { Magnitude } \\
\text { of applied } \\
\text { transformati } \\
\text { on has less } \\
\text { impact. }\end{array}$ & $\begin{array}{l}\text { 1. Scaling to } \\
\text { segmentatio } \\
\mathrm{n} \text { is difficult } \\
\text { as it requires } \\
\text { one class for } \\
\text { every patch } \\
\text { per image. }\end{array}$ \\
\hline 4 & $\begin{array}{l}\text { Intraretinal } \\
\text { and Sub } \\
\text { retinal } \\
\text { Fluid } \\
\text { segmentatio } \\
\text { n in } \\
\text { Longitudinal } \\
\text { Clinical } \\
\text { OCT using } \\
\text { SEAD } \\
\text { technique. }\end{array}$ & $\begin{array}{l}\text { 1. SEAD } \\
\text { with small } \\
\text { size can be } \\
\text { detected. } \\
\text { 2.Control } \\
\text { false } \\
\text { positives }\end{array}$ & $\begin{array}{l}\text { 1. Final } \\
\text { output relies } \\
\text { on layer } \\
\text { segmentatio } \\
\text { n results. } \\
2 \text {. The } \\
\text { absence of } \\
\text { smoothness } \\
\text { in limit, } \\
\text { which causes } \\
\text { issue in } \\
\text { precise } \\
\text { SEAD } \\
\text { outline. }\end{array}$ \\
\hline
\end{tabular}




\begin{tabular}{|c|c|c|c|}
\hline 5 & $\begin{array}{c}\text { Object } \\
\text { detection } \\
\text { using Faster } \\
\text { R-CNN }\end{array}$ & $\begin{array}{l}\text { 1. Cost } \\
\text { proficient } \\
\text { and a } \\
\text { successful } \\
\text { strategy for } \\
\text { object } \\
\text { recognition } \\
\text { as the RPN } \\
\text { improves } \\
\text { region } \\
\text { proposal } \\
\text { quality } \\
2 . \\
\text { Translation } \\
\text { invariant } \\
\text { property } \\
\text { decreased } \\
\text { the model } \\
\text { size. } \\
\text { 3. Multi } \\
\text { scale anchor } \\
\text { configuratio } \\
\text { n helps to } \\
\text { share } \\
\text { features } \\
\text { without } \\
\text { additional } \\
\text { expense for } \\
\text { scaling. } \\
\text { 4. A filter } \\
\text { pyramid or } \\
\text { image } \\
\text { pyramid to } \\
\text { anticipate } \\
\text { region of } \\
\text { various } \\
\text { manis } \\
\text { saves are } \\
\text { running } \\
\text { not required. }\end{array}$ & $\begin{array}{c}\text { 1. R-CNN's } \\
\text { exactness } \\
\text { relies upon } \\
\text { the } \\
\text { performance } \\
\text { of RPN } \\
\text { module. } \\
\text { 2. Faster R- } \\
\text { CNN } \\
\text { contains a } \\
\text { few } \\
\text { unshared } \\
\text { fully } \\
\text { connected } \\
\text { layers that } \\
\text { must be } \\
\text { computed } \\
\text { for every } \\
\text { one of } \\
\text { several } \\
\text { proposals. }\end{array}$ \\
\hline
\end{tabular}

\begin{tabular}{|c|c|c|c|}
\hline 6 & $\begin{array}{c}\text { OCT } \\
\text { Boundary } \\
\text { detection } \\
\text { using graph } \\
\text { cut approach }\end{array}$ & $\begin{array}{c}\text { 1. Even in } \\
\text { the existence } \\
\text { of pathology } \\
\text { and } \\
\text { morphologic } \\
\text { al changes, } \\
\text { this } \\
\text { algorithm } \\
\text { precisely and } \\
\text { productively } \\
\text { distinguishes } \\
\text { the retinal } \\
\text { layers } \\
\text { boundaries } \\
\text { from SD- } \\
\text { OCT } \\
\text { images. } \\
\text { 2.Search } \\
\text { spaces are } \\
\text { confined by } \\
\text { boundaries } \\
\text { and it gives } \\
\text { an } \\
\text { astounding } \\
\text { exactness } \\
\text { and shows } \\
\text { preferred } \\
\text { execution } \\
\text { over the } \\
\text { current } \\
\text { techniques }\end{array}$ & $\begin{array}{c}\text { 1. The error } \\
\text { for IZ (Inter- } \\
\text { digitations } \\
\text { Zone) and } \\
\text { RPE } \\
\text { (Retinal } \\
\text { pigment } \\
\text { epithelium) } \\
\text { boundary is } \\
\text { high due to } \\
\text { low contrast. }\end{array}$ \\
\hline 7 & $\begin{array}{c}\text { OCT } \\
\text { classification } \\
\text { using } \\
\text { MCME }\end{array}$ & $\begin{array}{l}\text { 1. Pre- } \\
\text { processing } \\
\text { requirements } \\
\text { are } \\
\text { minimum } \\
\text { for this } \\
\text { classification } \\
\text { approach. } \\
2 . \\
\text { Volumetric } \\
\text { OCT's } \\
\text { multi-slice } \\
\text { analysis is } \\
\text { possible. }\end{array}$ & $\begin{array}{l}\text { 1. MCME } \\
\text { model } \\
\text { requires a } \\
\text { prior } \\
\text { decompositi } \\
\text { on } \\
\text { 2. Result of } \\
\text { the model } \\
\text { depends on } \\
\text { each CNN } \\
\text { output as } \\
\text { several } \\
\text { classifier } \\
\text { networks are } \\
\text { combined in } \\
\text { this model. } \\
\text { 3. Splitting } \\
\text { of learned } \\
\text { feature space } \\
\text { by the } \\
\text { MCME } \\
\text { model } \\
\text { affects the } \\
\text { classification }\end{array}$ \\
\hline
\end{tabular}




\begin{tabular}{|c|c|c|c|}
\hline & & & rate. \\
\hline 8 & $\begin{array}{c}\text { OCT } \\
\text { segmentatio } \\
\text { n using FCN }\end{array}$ & $\begin{array}{l}\text { 1. Works on } \\
\text { any sized } \\
\text { input and } \\
\text { produces } \\
\text { output with } \\
\text { same spatial } \\
\text { dimensions. } \\
\text { 2. Pixel to } \\
\text { pixel } \\
\text { operation } \\
\text { streamlines } \\
\text { and } \\
\text { accelerates } \\
\text { learning and } \\
\text { inference. }\end{array}$ & $\begin{array}{l}\text { 1. The scale } \\
\text { of details in } \\
\text { upsampled } \\
\text { output is } \\
\text { restricted by } \\
\text { the network } \\
\text { prediction } \\
\text { stride }\end{array}$ \\
\hline 9 & $\begin{array}{c}\text { OCT } \\
\text { classification } \\
\text { using CNN }\end{array}$ & $\begin{array}{l}\text { 1. Different } \\
\text { surrogated } \\
\text { images } \\
\text { address the } \\
\text { requirement } \\
\text { of a } \\
\text { considerable } \\
\text { number of } \\
\text { images to } \\
\text { train CNN. } \\
\text { 2. In this } \\
\text { method, the } \\
\text { surrogate } \\
\text { image is } \\
\text { smaller than } \\
\text { original. } \\
\text { Thus the } \\
\text { memory } \\
\text { consumption } \\
\text { problem is } \\
\text { handled. }\end{array}$ & $\begin{array}{l}\text { 1. Volume } \\
\text { level } \\
\text { comparison } \\
\text { is time } \\
\text { consuming. }\end{array}$ \\
\hline
\end{tabular}

\begin{tabular}{|c|c|c|c|}
\hline 10 & $\begin{array}{c}\text { Retinal } \\
\text { image } \\
\text { classification } \\
\text { using CNN }\end{array}$ & $\begin{array}{l}\text { 1. Without } \\
\text { any image } \\
\text { pre- } \\
\text { processing } \\
\text { technique, } \\
\text { desired level } \\
\text { of } \\
\text { classification } \\
\text { can be } \\
\text { achieved. } \\
\text { 2. Attention } \\
\text { map learning } \\
\text { process can } \\
\text { be controlled } \\
\text { by training } \\
\text { LDN using } \\
\text { small } \\
\text { number of } \\
\text { OCT images } \\
\text { with } \\
\text { additional } \\
\text { annotation in } \\
\text { lesion level } \\
\text { 3. This } \\
\text { method can } \\
\text { detect } \\
\text { various } \\
\text { levels of } \\
\text { lesions. }\end{array}$ & $\begin{array}{l}\text { 1. Lesions of } \\
\text { Drusen with } \\
\text { small areas } \\
\text { are very } \\
\text { difficult to } \\
\text { detect. } \\
\text { 2. Miss- } \\
\text { classification } \\
\text { happens } \\
\text { between } \\
\text { both CNV } \\
\text { (Choroidal } \\
\text { Neovasculari } \\
\text { zation) and } \\
\text { DME as } \\
\text { both have } \\
\text { similar } \\
\text { visual } \\
\text { characteristi } \\
\text { cs. }\end{array}$ \\
\hline 11 & $\begin{array}{l}\text { Anomaly } \\
\text { detection } \\
\text { using } \\
\text { Bayesian U- } \\
\text { Net }\end{array}$ & $\begin{array}{l}\text { 1. Use of } \\
\text { uncertainty } \\
\text { is } \\
\text { incorporated } \\
\text { in automated } \\
\text { anomaly } \\
\text { detection. } \\
\text { 2. Instead of } \\
\text { manual } \\
\text { annotations, } \\
\text { weak labels } \\
\text { are utilized } \\
\text { for training. } \\
\text { So training } \\
\text { data can be } \\
\text { increased. }\end{array}$ & $\begin{array}{c}\text { 1. Post- } \\
\text { processing } \\
\text { algorithms } \\
\text { speculate } \\
\text { that } \\
\text { anomalous } \\
\text { regions are } \\
\text { areas } \\
\text { encompasse } \\
\text { d by } \\
\text { uncertainties } \\
\text {. So over- } \\
\text { segmentatio } \\
\text { n can } \\
\text { happen. }\end{array}$ \\
\hline
\end{tabular}

\section{CONCLUSION}

Various OCT segmentation and classification methods are analyzed. Each method has its own pros and cons. Various OCT image segmentation techniques and classification techniques are considered in this paper. Mostly OCT images are classified using supervised learning methods. As categorization of unlabelled bio markers requires more knowledge and expert opinion, few papers address the unsupervised method of OCT classification. 


\section{REFERENCES}

$1 \quad$ P. Seebock, Philipp Waldstein, Sebastian M. Klimscha, Sophie Bogunovic, Hrvoje Schlegl, Thomas Gerendas, Bianca S. Donner, Rene Schmidt-Erfurth, Ursula Langs, Georg, Unsupervised Identification of Disease Marker Candidates in Retinal OCT Imaging Data,IEEE Trans. Med. Imaging, vol. 38, no. 4, pp. 1037-1047, 2019.

Garvin, Mona Kathryn Abràmoff, Michael David Abràmoff, Michael David Wu, Xiaodong Russell, Stephen R. Burns, Trudy L. Sonka, Milan, Automated 3-D Intraretinal Layer Segmentation of Macular Spectral-Domain Optical Coherence Tomography Images, IEEE Trans. Med. Imaging, vol. 28, no. 9, pp. 1436-1447, 2009.

A. Dosovitskiy, P. Fischer, J. T. Springenberg, M. Riedmiller, and T. Brox, Discriminative unsupervised feature learning with exemplar convolutional neural networks, IEEE Trans. Pattern Anal. Mach. Intell., vol. 38, no. 9, pp. 1734 1747, 2016.

X. Xu, K. Lee, L. Zhang, M. Sonka, and M. D. Abràmoff, Stratified Sampling Voxel Classification for Segmentation of Intraretinal and Subretinal Fluid in Longitudinal Clinical OCT Data, IEEE Trans. Med. Imaging, vol. 34, no. 7, pp. 1616-1623, 2015.

S. Ren, K. He, R. Girshick, and J. Sun, Faster RCNN: Towards Real-Time Object Detection with Region Proposal Networks, IEEE Trans. Pattern Anal. Mach. Intell., vol. 39, no. 6, pp. 1137-1149, 2017.

M. A. Hussain, Md Akter Bhuiyan, Alauddin Turpin, Andrew Luu, Chi D. Smith, R. Theodore Guymer, Robyn H. Kotagiri, Ramamohanrao., Automatic identification of pathology-distorted retinal layer boundaries using SD-OCT imaging,IEEE Trans. Biomed. Eng., vol. 64, no. 7, pp. 1638-1649, 2017.

R. Rasti, H. Rabbani, A. Mehridehnavi, and F. Hajizadeh, Macular OCT Classification Using a Multi-Scale Convolutional Neural Network Ensemble, IEEE Trans. Med. Imaging, vol. 37, no. 4, pp. 1024-1034, 2018.

S. Shekkizhar and M. Lababidi, Tensorflow implementation of Fully Convolutional Networks for Semantic Segmentation, vol. 39, no. 4, pp. 640-651, 2017. assisted retinal OCT image classification based on convolutional neural networks,IEEE J. Biomed. Heal. Informatics, vol. 23, no. 1, pp. 253-263, 2019.

10 L. Fang, C. Wang, S. Li, H. Rabbani, X. Chen, and Z. Liu, Attention to Lesion: Lesion-Aware Convolutional Neural Network for Retinal Optical Coherence Tomography Image Classification,IEEE Trans. Med. Imaging, vol. 38, no. 8, pp. 1959-1970, 2019.

11 P. Seebock, Philipp Orlando, Jose Ignacio Schlegl, Thomas Waldstein, Sebastian M. Bogunovic, Hrvoje Klimscha, Sophie Langs, Georg Schmidt-Erfurth, Ursula, Exploiting Epistemic Uncertainty of Anatomy Segmentation for Anomaly Detection in Retinal OCT, IEEE Trans. Med. Imaging, vol. XX, no. Xx, pp. 1-1, 2019. 